\title{
Two Distinct Types of Hypercontractile Esophagus: Classic and Spastic Jackhammer
}

\author{
Yun Soo Hong, Yang Won Min, and Poong-Lyul Rhee \\ Department of Medicine, Samsung Medical Center, Sungkyunkwan University School of Medicine, Seoul, Korea
}

Hypercontractile esophagus (nicknamed jackhammer esophagus) is a recently defined disease within the esophageal motility disorders classification. Responses to treatments for jackhammer esophagus have been inconsistent in previous trials, possibly due to its heterogeneous manifestation. Thus, we reviewed 10 patients diagnosed with jackhammer esophagus and compared their clinical and manometric features at baseline. Additionally, manometric and symptomatic responses after treatment with known smooth muscle relaxants, including anticholinergic drugs (cimetropium bromide and scopolamine butylbromide) and a phosphodiesterase- 5 inhibitor (sildenafil) were compared. We observed two distinct subgroups in the findings: one with hypercontractility and normal distal latencies ("classic jackhammer esophagus," $n=7$ ) and the other with hypercontractility and short distal latencies ("spastic jackhammer esophagus," $n=3$ ). The two types also differed in their responses to medications in that symptoms improved upon treatment with an anticholinergic agent in classic jackhammer esophagus patients, while spastic jackhammer esophagus was unresponsive to both the anticholinergic drugs and the phosphodiesterase- 5 inhibitor. In conclusion, hypercontractile esophagus may be a heterogeneous disease with different underlying pathophysiologies. We introduced two novel terms, "classic jackhammer esophagus" and "spastic jackhammer esophagus," to distinguish the two types. (Gut Liver 2016;10:859-863)

Key Words: Deglutition disorders; Dysphagia; Esophageal motility disorders

\section{INTRODUCTION}

Nutcracker esophagus was defined as high-amplitude peristaltic contraction of the distal esophagus (amplitude $>180 \mathrm{~mm}$
$\mathrm{Hg}$ ) on conventional manometry. ${ }^{1}$ The revised Chicago classification introduced distal contractile integral (DCI) using highresolution manometry (HRM) to reflect contractile vigor and defined hypercontractile esophagus (jackhammer esophagus [JE]), as at least one contraction with DCI >8,000 $\mathrm{mm} \mathrm{Hg} \cdot s \cdot \mathrm{cm}$. ${ }^{2}$ Since it is a value unobserved in normal subjects and is usually accompanied by esophageal symptoms, such as dysphagia and chest pain, ${ }^{3}$ this new definition seemed clinically more relevant. However, the diagnosis of JE is usually made solely based on a manometric criterion, while previous studies reported diverse clinical manifestations and treatment responses, suggesting the possibility of JE being a heterogeneous disease entity with complex pathophysiology. ${ }^{4-7}$ Because identification and understanding of different phenotypes and pathophysiology can enhance treatment outcomes, we describe manometric features, clinical manifestations and treatment responses of 10 patients who were diagnosed with and treated for JE. Additionally, we introduce two types of JE based on these findings.

\section{CASE REPORT}

We reviewed 10 patients diagnosed with JE according to the revised Chicago classification ${ }^{2}$ at Samsung Medical Center between November 2011 and April 2014. Patients underwent upper endoscopy, esophagography, esophageal HRM, and 24hour impedance-pH (24h Imp-pH) monitoring. Esophagography was performed with barium sulfate (Solotop; Taejoon Pharm, Seoul, Korea) of 140\% weight in volume. HRM (Sandhill Scientific, Highlands Ranch, CO, USA) was performed in the standard fashion. ${ }^{2}$ For $24 \mathrm{~h}$ Imp-pH monitoring, a single-use, combined impedance and $\mathrm{pH}$ probe attached to a portable data logger (Sandhill Scientific) was used. BioView MII software (Sandhill Scientific) was used for data analysis.

Upon the diagnosis of JE, HRM was repeated after adminis-

Correspondence to: Poong-Lyul Rhee

Department of Medicine, Samsung Medical Center, Sungkyunkwan University School of Medicine, 81 Irwon-ro, Gangnam-gu, Seoul 06351, Korea

Tel: +82-2-3410-3409, Fax: +82-2-3410-6983, E-mail: plrhee@skku.edu

Received on August 10, 2015. Revised on November 9, 2015. Accepted on February 2, 2016. Published online July 27, 2016

pISSN 1976-2283 eISSN 2005-1212 http://dx.doi.org/10.5009/gnl15388

@ This is an Open Access article distributed under the terms of the Creative Commons Attribution Non-Commercial License (http://creativecommons.org/licenses/by-nc/4.0) which permits unrestricted non-commercial use, distribution, and reproduction in any medium, provided the original work is properly cited. 
tration of either sildenafil (Viagra $100 \mathrm{mg}$; per oral [PO]; Pfizer, New York, NY, USA) or cimetropium bromide (Algiron $5 \mathrm{mg}$; intravenous; Boehringer Ingelheim $\mathrm{GmbH}$, Ingelheim, Germany). Being a phosphodiesterase- 5 inhibitor ${ }^{7}$ and an anticholinergic agent, ${ }^{5}$ respectively, their potential effects on smooth muscle relaxation and symptom relief had been suggested in previous studies. Sildenafil was used until March 2012 but cimetropium took its place since November 2011, with an overlapping period. HRM was performed 30 minutes after sildenafil ingestion or 5 minutes after cimetropium injection. A positive manometric response was defined as a decrease in DCI to normal level (DCI $<5,000 \mathrm{~mm} \mathrm{Hg} \cdot \mathrm{s} \cdot \mathrm{cm})$. When the response was positive on HRM with sildenafil or cimetropium, sildenafil $(100 \mathrm{mg}$, once daily, P0) or scopolamine butylbromide (10 mg, three times a day before meals, P0; Boehringer Ingelheim), another antimuscarinic anticholinergic drug, was prescribed as maintenance therapy, respectively. Symptom improvement was evaluated after treatment for 1 or 2 months.

Six of the 10 patients were male and the median age was 64 years (range, 46 to 76 years). Dysphagia and chest pain were the two most common symptoms for undergoing manometric evaluation. On upper endoscopy, one case of erosive esophagitis (LA classification A) was found. Esophagography showed corkscrew appearance in one patient. In two other cases, abnormal tertiary movement and tortuous peristalsis of the distal esophagus was noted respectively, but neither was diagnostic for any specific esophageal motility disorder. On HRM, the median value of maximum DCI was 13,826 mm Hg.s.cm (range, 8,607 to $38,749 \mathrm{~mm} \mathrm{Hg} \cdot \mathrm{s} \cdot \mathrm{cm}$ ) and the median distal latency (DL) was 5.4 seconds (range, 2.0 to 7.0 seconds). Three patients had premature contraction, defined as DL $<4.5$ seconds in $20 \%$ or greater swallows. ${ }^{2}$ The median integrated relaxation pressure (IRP) was $19 \mathrm{~mm} \mathrm{Hg}$ (range, 5.3 to $34 \mathrm{~mm} \mathrm{Hg}$ ). In the six patients with IRP >15 mm Hg, propagating peristalsis and lower esophageal sphincter (LES) relaxation were well preserved on the esophageal pressure topography. In addition, neither passage disturbance nor esophageal dilatation was seen on the esophagography. Therefore, achalasia was considered less likely despite high IRP values.

Among the 10 patients, we noticed a distinct group of three patients with features of both hypercontractility and spasticity (patients number 8, 9, and 10 in Table 1). We called this group with coexistent hypercontractility and premature contraction "spastic JE" to distinguish it from "classic JE," which conveys only hypercontractility according to Chicago classification. Patients were divided into classic JE $(n=7)$ and spastic JE $(n=3)$ groups and were compared with each other. Fig. $1 \mathrm{~A}$ and $\mathrm{B}$ shows representative HRM findings for each group. Clinical features were not different between the two groups and dysphagia was the most common symptom in both groups (four and two in classic JE and spastic JE, respectively). In addition, parameters of HRM other than DL were not statistically different between the two groups. HRM findings after administration of medications in classic JE and spastic JE are shown in Fig. 1C and D, respectively. Manometric responses were positive with the use of either cimetropium bromide or sildenafil in all patients (Table 1). Scopolamine as maintenance treatment improved symptoms in three of the four (75\%) classic JE patients, while one (50\%) spastic JE patient responded. Sildenafil was effective in one (33.3\%) classic JE patient, but was ineffective in the one spastic JE patient prescribed with sildenafil. In summary, classic JE showed a better response to anticholinergics, whereas spastic JE was controlled less effectively by either anticholinergics or phosphodiesterase- 5 inhibitor.

\section{DISCUSSION}

$\mathrm{JE}$ is a rare motility disorder presenting with dysphagia and/

Table 1. Summary of 10 Patients with Jackhammer Esophagus

\begin{tabular}{|c|c|c|c|c|c|c|c|}
\hline \multirow[b]{2}{*}{ No. } & \multirow[b]{2}{*}{ Type of JE } & \multirow[b]{2}{*}{ Symptom } & \multicolumn{2}{|c|}{ HRM parameter } & \multirow[b]{2}{*}{ Medication } & \multicolumn{2}{|c|}{ Response to medication } \\
\hline & & & DL, sec & $\begin{array}{c}\text { Maximum DCI, } \\
\mathrm{mm} \mathrm{Hg} \cdot \mathrm{s} \cdot \mathrm{cm}\end{array}$ & & Manometric & Symptomatic \\
\hline 1 & Classic JE & Dysphagia, regurgitation & 7.7 & 38,074 & Cimetropium & $(+)$ & $(+)$ \\
\hline 2 & & Globus sense, hoarseness & 6.4 & 9,901 & Cimetropium & $(+)$ & $(+)$ \\
\hline 3 & & Dysphagia, chest pain & 5.4 & 8,607 & Cimetropium & $(+)$ & $(+)$ \\
\hline 4 & & Chest pain & 6.1 & 13,430 & Cimetropium & $(+)$ & $(-)$ \\
\hline 5 & & Heartburn, dysphagia & 5.3 & 17,888 & Sildenafil & $(+)$ & $(+)$ \\
\hline 6 & & Dysphagia, chest pain & 5.4 & 14,221 & Sildenafil & $(+)$ & $(-)$ \\
\hline 7 & & Dysphagia, epigastric discomfort & 7.5 & 12,394 & Sildenafil & $(+)$ & $(-)$ \\
\hline 8 & Spastic JE & Chest pain, heartburn & 4.2 & 10,611 & Cimetropium & $(+)$ & $(+)$ \\
\hline 9 & & Dysphagia, globus sense & 4.5 & 20,570 & Cimetropium & $(+)$ & $(-)$ \\
\hline 10 & & Dysphagia, regurgitation & 2.0 & 38,749 & Sildenafil & $(+)$ & $(-)$ \\
\hline
\end{tabular}

JE, jackhammer esophagus; HRM, high-resolution manometry; DL, distal latency; DCI, distal contractile integral. 

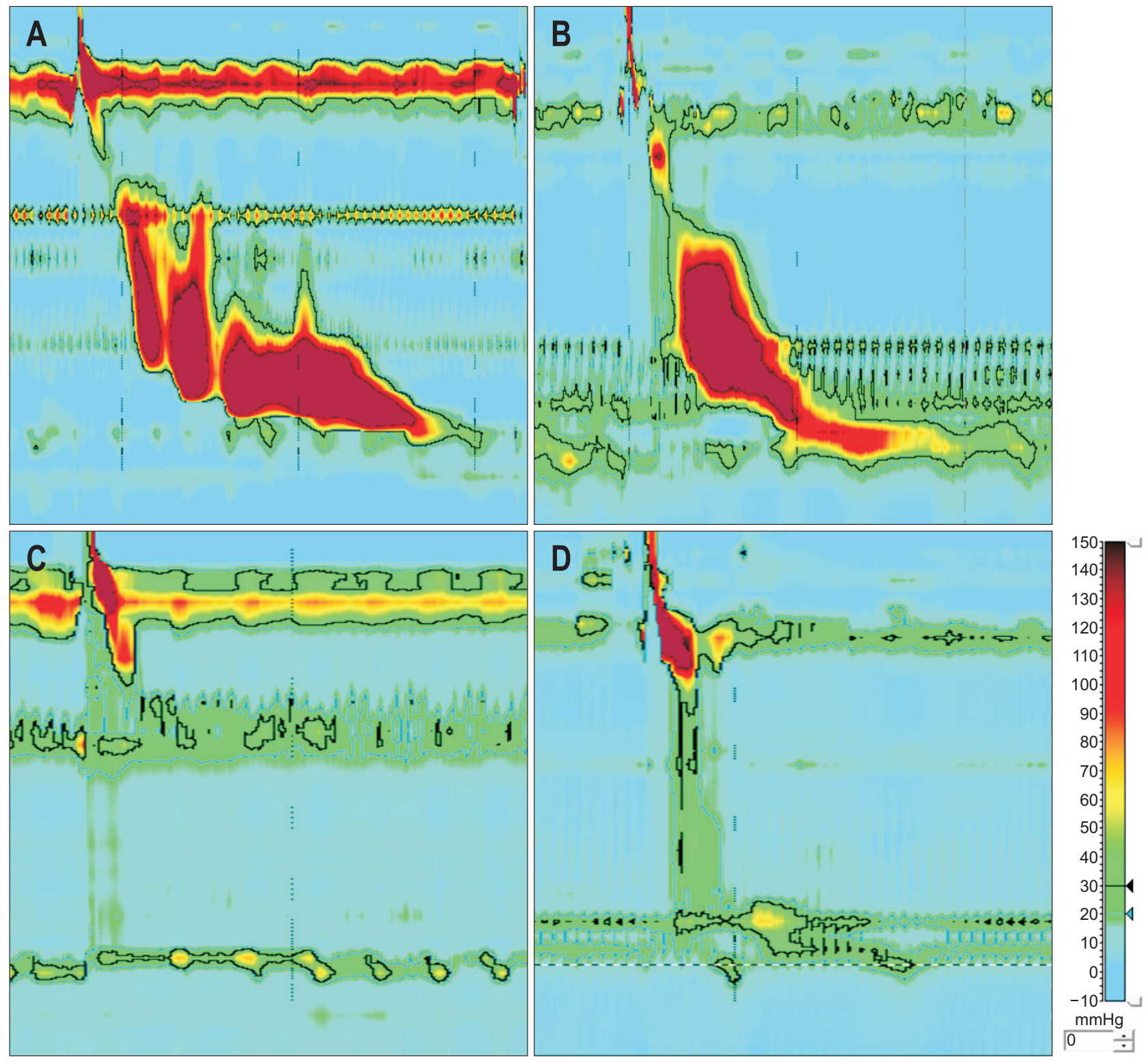

Fig. 1. (A) A typical finding of classic jackhammer esophagus (JE) with normal distal latency on esophageal high-resolution manometry (HRM). (B) A typical finding of spastic JE with reduced distal latency on esophageal HRM. (C) Manometric response 30 minutes after sildenafil (100 mg) ingestion in a classic JE patient. (D) Manometric response 5 minutes after cimetropium bromide (5 mg) injection in a spastic JE patient.

or chest pain. It is characterized by hypercontraction of the esophageal smooth muscle. ${ }^{2}$ Excessive cholinergic drive or muscle hypertrophy is considered responsible for such high amplitude contraction. ${ }^{8}$ For this reason, smooth muscle relaxants, including calcium channel blockers, ${ }^{4}$ atropine,${ }^{5}$ botulinum toxin, ${ }^{6}$ and sildenafil ${ }^{7}$ have often been chosen as treatment options. In these numerous trials, the results were not consistent enough to establish a consensus. Also, even the most recently published literature on the treatment for hypercontractile or spastic esophageal motility disorders included heterogeneous groups of diseases, ${ }^{6}$ and a study consisting only of JE defined by the Chicago classification has not been reported. Thus, we analyzed patients who met the criteria for JE based on the Chicago clas- sification and grouped them based on the presence of spasticity (DL $<4.5$ seconds) and hypercontractility. They differed not only in the presence of premature contraction but also in the treatment response to medications known to relieve smooth muscle contraction.

DL reflects the duration of deglutitive inhibition, governed by inhibitory postganglionic neurons. ${ }^{9}$ With nitric oxide being the dominant neurotransmitter, ${ }^{10,11}$ impairment in the nitrergic pathway is responsible for premature contraction, or spasm, of the lower esophagus. On the other hand, contractile vigor of the distal esophageal contraction, represented by DCI, is a consequence of cholinergic excitation. ${ }^{12}$ In accordance with the theory, JE with normal DL (classic JE) was responsive to anti- 
cholinergic agents, while JE with premature contraction (spastic JE) was not effectively treated with an anticholinergic drug or sildenafil alone. From these observations, the current classification of JE seems to consist of two subgroups with distinct underlying pathophysiologic mechanisms within the category of hypercontractile esophagus.

Premature contraction is also observed in other spastic conditions; type III achalasia and distal esophageal spasm (DES). ${ }^{13}$ Although hypercontractility can be accompanied in type III achalasia, ${ }^{9}$ its predominant feature is abnormal relaxation of the LES and absent peristalsis. Even though IRP exceeded 15 $\mathrm{mm} \mathrm{Hg}$ in six patients, LES relaxation and peristalsis were well preserved in other exams. Also, IRP is a complex metric, which can potentially be affected by multiple factors. IRP tends to increase with a premature distal contraction ${ }^{14}$ and cutoff values varies among manometric devices. ${ }^{15}$ DES, on the other hand, is characterized by contractions of normal IRP and reduced DL regardless of contractility. While DES is mainly of simultaneous contraction, spastic JE is a phenotype of both hypercontraction and simultaneous contraction. In short, these diseases may fall into a spectrum of an imbalance between inhibitory and excitatory neuronal function depending on the degree.

In these cases, although all patients had positive manometric response, response to maintenance treatment varied among them. In other words, the presence of manometric improvement did not indicate long-term symptomatic improvement. One plausible explanation would be different pharmacokinetics and pharmacodynamics of cimetropium bromide and scopolamine butylbromide. Although both are categorized as antimuscarinic anticholinergic drug, one is an intravenous form and the other is PO form with bioavailability of $100 \%$ and $8 \%$, respectively. ${ }^{16}$ Also, different drug compositions would result in distinct mechanisms of action. Future studies using premedication with PO scopolamine, rather than intravenous cimetropium, may minimize the discordance. For sildenafil, once-daily dose may not have been sufficient to relieve an on-going or postprandial esophageal symptoms due to the rapid onset of action within 0.5 to 1 hour and short plasma half-life of about 4 hours. ${ }^{17}$ We may expect better outcome with an extended-release form of phosphodiesterase- 5 inhibitor, such as Tadalafil, with a half-life of 17.5 hours, ${ }^{18}$ when it becomes available to disease other than pulmonary hypertension and erectile dysfunction. Additionally, for spastic JE, the combination of an anticholinergic agent with sildenafil could be considered as another therapeutic option.

In conclusion, hypercontractile esophagus, may be a heterogeneous disease entity with distinct features. Here, we introduce new terminologies, classic JE and spastic JE, to better represent underlying pathophysiology. Further large scale prospective studies are warranted to distinguish esophageal motility disorders with excessive contractile features and to properly manage these patients.

\section{CONFLICTS OF INTEREST}

No potential conflict of interest relevant to this article was reported.

\section{REFERENCES}

1. Spechler SJ, Castell DO. Classification of oesophageal motility abnormalities. Gut 2001;49:145-151.

2. Bredenoord AJ, Fox M, Kahrilas PJ, et al. Chicago classification criteria of esophageal motility disorders defined in high resolution esophageal pressure topography. Neurogastroenterol Motil 2012;24 Suppl 1:57-65.

3. Roman S, Pandolfino JE, Chen J, Boris L, Luger D, Kahrilas PJ. Phenotypes and clinical context of hypercontractility in high-resolution esophageal pressure topography (EPT). Am J Gastroenterol 2012;107:37-45.

4. Cattau EL Jr, Castell DO, Johnson DA, et al. Diltiazem therapy for symptoms associated with nutcracker esophagus. Am J Gastroenterol 1991;86:272-276.

5. Korsapati H, Bhargava V, Mittal RK. Reversal of asynchrony between circular and longitudinal muscle contraction in nutcracker esophagus by atropine. Gastroenterology 2008;135:796-802.

6. Marjoux S, Brochard C, Roman S, et al. Botulinum toxin injection for hypercontractile or spastic esophageal motility disorders: may high-resolution manometry help to select cases? Dis Esophagus 2015;28:735-741

7. Lee JI, Park H, Kim JH, Lee SI, Conklin JL. The effect of sildenafil on oesophageal motor function in healthy subjects and patients with nutcracker oesophagus. Neurogastroenterol Motil 2003;15:617-623.

8. Dogan I, Puckett JL, Padda BS, Mittal RK. Prevalence of increased esophageal muscle thickness in patients with esophageal symptoms. Am J Gastroenterol 2007;102:137-145.

9. Kahrilas PJ, Boeckxstaens G. The spectrum of achalasia: lessons from studies of pathophysiology and high-resolution manometry. Gastroenterology 2013;145:954-965.

10. Goyal RK, Chaudhury A. Physiology of normal esophageal motility. J Clin Gastroenterol 2008;42:610-619.

11. Conklin JL. Nitric oxide: a mediator of esophageal motor function. J Lab Clin Med 1998;131:10-20.

12. Gyawali CP, Bredenoord AJ, Conklin JL, et al. Evaluation of esophageal motor function in clinical practice. Neurogastroenterol Motil 2013;25:99-133.

13. Roman S, Lin Z, Pandolfino JE, Kahrilas PJ. Distal contraction latency: a measure of propagation velocity optimized for esophageal pressure topography studies. Am J Gastroenterol 2011;106:443451.

14. Lin Z, Kahrilas PJ, Roman S, Boris L, Carlson D, Pandolfino JE. Refining the criterion for an abnormal Integrated Relaxation Pressure in esophageal pressure topography based on the pattern of esophageal contractility using a classification and regression tree 
model. Neurogastroenterol Motil 2012;24:e356-e363.

15. Roman S, Gyawali CP, Xiao Y, Pandolfino JE, Kahrilas PJ. The Chicago classification of motility disorders: an update. Gastrointest Endosc Clin N Am 2014;24:545-561.

16. Samuels LA. Pharmacotherapy update: hyoscine butylbromide in the treatment of abdominal spasms. Clin Med Ther 2009;1:647655.
17. Nichols DJ, Muirhead GJ, Harness JA. Pharmacokinetics of sildenafil after single oral doses in healthy male subjects: absolute bioavailability, food effects and dose proportionality. Br J Clin Pharmacol 2002;53 Suppl 1:5S-12S.

18. Gupta M, Kovar A, Meibohm B. The clinical pharmacokinetics of phosphodiesterase- 5 inhibitors for erectile dysfunction. J Clin Pharmacol 2005;45:987-1003. 